\title{
Preparation and Characterization of Vancomycin Hydrochloride-Loaded Mesoporous Silica Composite Hydrogels
}

\begin{abstract}
Ming Sun ${ }^{1,2}$, Lidi Cheng ${ }^{1,2}$, Zexian $X u^{1,2}$, Liqiang Chen ${ }^{1,2,3}$, Yanshan Liu ${ }^{1,2,3}$, Yaoxiang $X u^{1,2}$ Dongyang Zhou ${ }^{1,2}$, Xiuxiu Zhang ${ }^{1,2}$, Qihui Zhou ${ }^{1,2,4 *}$ and Jian Sun ${ }^{1,2,3,5 *}$

${ }^{1}$ The Affiliated Hospital of Qingdao University, Qingdao, China, ${ }^{2}$ School of Stomatology of Qingdao University, Qingdao, China, ${ }^{3}$ Dental Digital Medicine \& 3D Printing Engineering Laboratory of Qingdao, Qingdao, China, ${ }^{4}$ Institute for Translational Medicine, The Affiliated Hospital of Qingdao University, Qingdao, China, ${ }^{5}$ Shandong Provincial Key Laboratory of Digital Medicine and Computer-Assisted Surgery, Qingdao, China
\end{abstract}

This study aims to explore the feasibility of the novel temperature-sensitive hydrogel-based dual sustained-release system (Van/SBA-15/CS-GP-SA) in the repair and treatment of infectious jaw defects. Van/SBA-15 was prepared using the mesoporous silica (SBA-15) as a carrier for vancomycin hydrochloride (Van), and Van/SBA-15 was characterized by scanning electron microscopy (SEM), transmission electron microscopy (TEM), energy dispersive spectrometry (EDS), X-ray photoelectron spectroscopy (XPS), Fourier transform infrared (FTIR), Brunauer-Emmett-Teller (BET), and Barrett-Joyner-Halenda (BJH). The characterization results confirm that Van is loaded in SBA-15 successfully. Van/SBA-15/ CS-GP-SA is constructed by encapsulating Van/SBA-15 in chitosan-sodium glycerophosphate-sodium alginate hydrogel (CS-GP-SA). The microstructures, sustained-release ability, biocompatibility, and antibacterial properties of Van/SBA-15/ CS-GP-SA were systematically studied. Van/SBA-15/CS-GP-SA is found to have promising sustained-release ability, outstanding biocompatibility, and excellent antibacterial properties. This study provides new ideas for the management of infectious jaw defects.

Keywords: biomaterials, hydrogel, drug delivery, biocompatibility, antibacterial

\section{INTRODUCTION}

The number of cases of jaw defects caused by accidents, trauma, cancer, congenital malformations, and other related diseases is increasing, with infected bone defects caused by wound contamination being a special type of bone defect (Darwich et al., 2021; Khorasani et al., 2021; Wang et al., 2021b). Until now, repairing infected jaw defects has remained a challenge due to its high recurrence rate for oral and maxillofacial surgeons (Zhang et al., 2019). To our knowledge, infection is due to the local presence of pathogenic bacteria in the lesion, and once an infected jaw defect occurs, it may require multiple surgical debridements and long-term systemic antibiotic therapy, which may lead to the development of secondary injury, adverse antibiotic reactions, or lifelong functional impairment, and increase the financial burden on the health-care system (Vestby et al., 2020; Wang et al., 2020). Therefore, antimicrobial drug carriers, a topical strategy, have become an adjunct to the prevention and treatment of infections in jaw defects (Alazzawi et al., 2021; Han et al., 2021). Topical 
administration at or near the site of infection diminishes the toxic effects of antibacterial agents, reduces the required dose of antibiotics, and enhances the bioavailability and safety of the drug (Wychowański et al., 2021; Zheng et al., 2021).

At present, vancomycin hydrochloride (Van), a glycopeptide antibiotic, has been widely used to treat infectious jaw defects (Ahadi et al., 2019). However, due to the short half-life of Van, patients need to be injected with it repeatedly, which may cause adverse effects in the organism (Pecci et al., 2020). SBA-15, a type of ordered mesoporous silica material with a two-dimensional hexagonal pore structure, is a promising carrier for the controlled release of therapeutic agents (Alkafajy and Albayati, 2020; Esperanza Adrover et al., 2020; Seljak et al., 2020; Sun et al., 2019). Large pore volume, size, and high surface area of SBA-15 contribute to an improved drug loading rate (Bavnhøj et al., 2019; Alkafajy and Albayati, 2020). Its mesoporous structure helps improve drug stability and prolong drug release time (Moritz and Marek, 2012; Shen et al., 2020). Hydrogels are also widely used as carriers in drug release because of their ability to modulate the rate of drug release effectively (Zhao et al., 2020). Chitosan (CS) has antibacterial properties, excellent cytoadhesive properties, biocompatibility, and stability (Abinaya et al., 2019; Li et al., 2020; Cui et al., 2021). The positive charge on CS can inhibit the growth and reproduction of some bacteria and viruses, which is beneficial to reduce the infection (Abid et al., 2019; Hao et al., 2021; Yin et al., 2021). The three-dimensional network structure of chitosan temperature-sensitive hydrogels can provide a good microenvironment for cell proliferation, migration, and differentiation and can guide the long entry of host cells (Zhao et al., 2020; Kang et al., 2021; Sordi et al., 2021; Yang et al., 2021). Sodium alginate (SA) is a natural polysaccharide that is a byproduct of iodine and mannitol extraction from the brown algae kelp or sargassum, which has promising biocompatible and biodegradable (Mujtaba and Alotaibi, 2020). Studies have shown that CS forms a cross-linked structure with SA through electrostatic interaction and hydrogen bonding, which strengthens the linkage between the molecular chains of sodium alginate, thus enhancing the mechanical properties and release control ability of the hydrogel (Kiti and Suwantong, 2020; Li et al., 2020; Kayan and Kayan, 2021).

To explore the feasibility of the dual sustained-release hydrogel system in the repair and treatment of infectious jaw defects, Van/SBA-15/CS-GP-SA was prepared and characterized.

\section{MATERIALS AND METHODS}

\subsection{Materials}

Van and SA were purchased from Dalian Meilun Biotechnology Co., Ltd. (Dalian, China). SBA-15 was provided by Nanjing XFNANO Materials Tech Co., Ltd. CS (deacetylation degree $\geq 95 \%$ ) and acetic acid (purity $\geq 99.8 \%$ ) were obtained from Shanghai Macklin Biochemical Co., Ltd. (Shanghai, China).

\subsection{Preparation of Van/SBA-15}

Hundred milligrams of SBA-15 was dissolved in $10 \mathrm{ml}$ of doubledistilled water and then sonicated to obtain SBA-15 suspension.
Van powder $(20 \mathrm{mg})$ was added to the SBA-15 suspension, and the mixture was stirred for $8 \mathrm{~h}$ at room temperature. Further $2 \mathrm{~h}$ of vacuum treatment was then carried out in a vacuum oven to facilitate the encapsulation of the Van in SBA-15. Subsequently, the mixture was centrifuged $(8,000 \mathrm{rpm}, 10 \mathrm{~min})$ and washed twice with double-distilled water. The resultant Van/SBA-15 was stored at $4^{\circ} \mathrm{C}$. Meanwhile, to confirm the drug encapsulation efficiency, the supernatant and wash solution were collected and analyzed at a wavelength of $280 \pm 2 \mathrm{~nm}$ using an ultraviolet-visible (UV) spectrophotometer (Aucybest, China). The encapsulation efficiency of Van/SBA-15 was $36.8 \%$.

\subsection{Characterization of Van/SBA-15}

SBA-15 and Van/SBA-15 were characterized by SEM (JSM7001F, Japan) and TEM (Jeol/JEM 2100, USA). For SEM observation, samples were sputter-coated with gold to increase electronic conductivity. And TEM images were taken with a JEOL JEM 2100 electron microscope at an accelerating voltage of $200 \mathrm{kV}$. The elemental profiles were analyzed by EDS (IXRF550i, USA).

The element compositions were detected by XPS (ESCALAB 250XI, USA). FTIR spectra of SBA-15 and Van/SBA-15 were measured using the $\mathrm{KBr}$ pellet method on a Nicolet 6700 spectrometer (Thermo, USA) from 4,000 to $400 \mathrm{~cm}^{-1}$. The $\mathrm{BET}$ and $\mathrm{BJH}$ analyses were employed to determine the pore size distribution, pore diameter, and specific surface area, through $\mathrm{N}_{2}$ adsorption-desorption isotherms (V-Sorb 2800P analyzer, Gold APP, China).

\subsection{Preparation and Characterization of Van/SBA-15/CS-GP-SA}

Sodium glycerophosphate (GP, $600 \mathrm{mg}$ ) was dissolved in $1 \mathrm{ml}$ of double-distilled water. After that, $40 \mathrm{mg}$ of SA was added to the solution. The resultant GP-SA solution was refrigerated at $4^{\circ} \mathrm{C}$. Chitosan $(250 \mathrm{mg}$ ) was dissolved in $10 \mathrm{ml}$ of $0.1 \mathrm{~mol} / \mathrm{L}$ acetic acid and then stirred for $2 \mathrm{~h}$. GP-SA and a certain amount of Van/ SBA-15 were added to the pre-prepared chitosan solution and then stirred for $30 \mathrm{~min}$. The obtained sol was poured into a mold soaked in a $37^{\circ} \mathrm{C}$ water bath to produce Van/SBA-15/CS-GP-SA gel (Laurano et al., 2020). The morphology of CS-GP-SA, Van/ CS-GP-SA, and Van/SBA-15/CS-GP-SA were observed by SEM and TEM.

\subsection{Investigation of In Vitro Drug Release}

In vitro drug release was analyzed by using the dialysis method (Ma and Shi, 2019). Van standard solution was diluted to different concentrations $(200-1,000 \mu \mathrm{g} / \mathrm{ml})$, and the absorbance at $280 \pm 2 \mathrm{~nm}$ of the solutions was measured. The standard curve was constructed by plotting concentration against absorbance. Each Van/SBA-15, Van/CS-GP-SA, and Van/SBA-15/CS-GP-SA was weighed to $200 \mathrm{mg}$ and then placed in a dialysis bag. The dialysis bag was placed in a beaker containing a release medium ( $10 \mathrm{ml}$ of $\mathrm{PBS}, \mathrm{pH}=7.4$ ), sealed, and then shaken at a constant speed at $37^{\circ} \mathrm{C}$. The release solution $(3 \mathrm{ml})$ was collected at different time points and stored until further tests, while equal amounts of PBS were supplemented to the solution. UV 



FIGURE 1 | SEM images of SBA-15 (A) and Van/SBA-15 (D), TEM images of SBA-15 (B) and Van/SBA-15 (E), EDS analysis of SBA-15 (C), and Van/SBA-15 (F).

absorbance at $280 \pm 2 \mathrm{~nm}$ was monitored. The concentrations were calculated based on the standard curves, and the cumulative release of Van at different time points was calculated.

\subsection{Analysis of In Vitro Cytocompatibility}

The samples used in this experiment were divided into four groups: control, CS-GP-SA, Van/CS-GP-SA, and Van/SBA-15/ CS-GP-SA. The hydrogels were sterilized under ultraviolet light for $2 \mathrm{~h}$ immediately after manufacture and then immersed in a cell culture medium at a surface area of $1 \mathrm{ml} / \mathrm{cm}^{2}$ of hydrogel for $24 \mathrm{~h}$ at $37^{\circ} \mathrm{C}$ to extract the compounds. The MT3C3-E1 cells were seeded in 96-well plates at $5 \times 10^{3}$ cells/well. Five replicate samples in each group were prepared. The plates were incubated at $37^{\circ} \mathrm{C}$ in an incubator saturated with $5 \% \mathrm{CO}_{2}$ for $24 \mathrm{~h}$ (Ji et al., 2021). After the cells adhered to the bottom of the well, the supernatant was discarded. Then the extract $(200 \mu \mathrm{l})$ was added to the CS-GP-SA, Van/CS-GP-SA, and Van/SBA-15/CSGP-SA group, and the cell culture medium at the same volume was added to the control group. After continuously incubated for 1,3 , and $5 \mathrm{~d}$, the numbers of viable cells were counted using the Cell Counting Kit-8 (CCK-8) (Sigma). CCK-8 solution $(20 \mu \mathrm{l})$ was added to each well, and the absorbance at $450 \mathrm{~nm}$ was measured after $1 \mathrm{~h}$. For the quantification of cell number, 4,6diamidino-2-phenylindole (DAPI) was used to stain the cell nuclei (Pierozan and Karlsson, 2019).

\subsection{Analysis of In Vitro Antibacterial Activity}

The hydrogel was sterilized under ultraviolet light for $2 \mathrm{~h}$ before use (Op't Veld et al., 2020). Then $200 \mu \mathrm{l}$ suspension of Escherichia coli (E. coli) and Staphylococcus aureus (S. aureus) were spread evenly on a Luria-Bertani (LB) agar plate and then incubated $37^{\circ} \mathrm{C}$ for $1 \mathrm{~h}$. CS-GP-SA, Van/CS-GP-SA, and Van/SBA-15/CSGP-SA with a diameter of $1 \mathrm{~cm}$ were placed on the $\mathrm{LB}$ agar plate containing the bacteria and then incubated at $37^{\circ} \mathrm{C}$ for another
$24 \mathrm{~h}$ in a bacterial incubator; Van/CS-GP-SA and Van/SBA-15/ CS-GP-SA contain an equal amount of vancomycin. After that, the diameters of the inhibition zones were measured. The data were the average of the three replicate samples.

\subsection{Statistical Analysis}

Statistical analysis was conducted using SPSS version 26.0. Oneway ANOVA and Tukey's multiple comparison method were used to determine the significant differences between groups. The differences with $p<0.05$ were considered statistically significant.

\section{RESULTS AND DISCUSSION}

\subsection{Characteristics of Van/SBA-15}

The SEM image showed that SBA-15 microscopically consisted of many short cylindrical rod-shaped particles with relatively uniform sizes, which is the typical morphological feature of SBA-15 molecular sieves. The surface morphologic image of Van/SBA-15 clearly showed that Van was adsorbed on the surface of SBA-15, which is an indication that the surface of SBA-15 has the capacity to adsorb drugs (Figures 1A,D). The TEM image showed that SBA-15 had a two-dimensional hexagonal structure. The pores of Van/SBA-15 became blurred, suggesting that Van was successfully loaded into SBA-15 (Figures 1B,E). The EDS spectrum showed that SBA-15 contained $\mathrm{O}$ and $\mathrm{Si}$, and Van/ SBA-15 contained not only $\mathrm{O}$ and $\mathrm{Si}$ but also $\mathrm{N}$ and $\mathrm{Cl}$ (Figures 1C,F). Van/SBA-15 contained two elements, $\mathrm{N}$ and $\mathrm{Cl}$, which are not present in SBA-15, and these two elements are formally Van's elements. These EDS results further verify the TEM result.

Equally, the XPS results showed that Van/SBA-15 powder exhibited the N1s peak at $400.3 \mathrm{eV}$ and $\mathrm{Cl} 2 \mathrm{p}$ peak at $201.6 \mathrm{eV}$, 

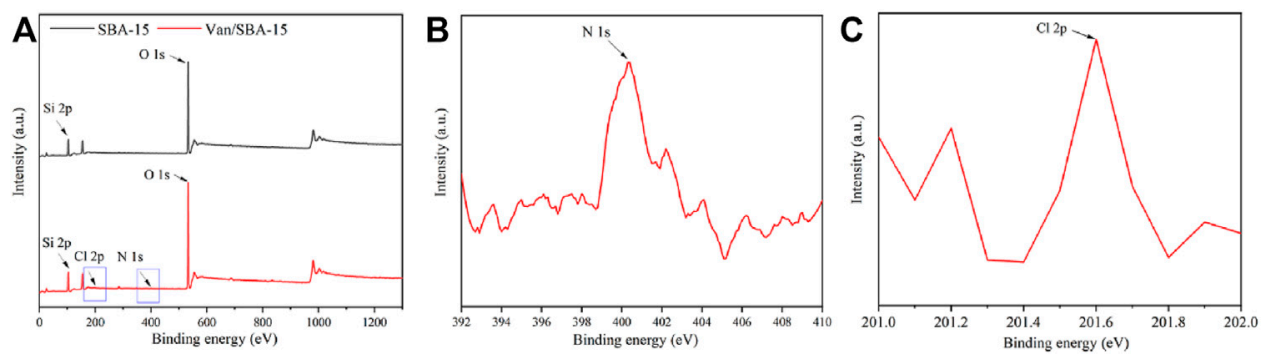

FIGURE 2 |XPS images of SBA-15 and Van/SBA-15 (A), XPS image of the N1s peak of Van/SBA-15 (B), and XPS image of the Cl2p peak of Van/SBA-15 (C).

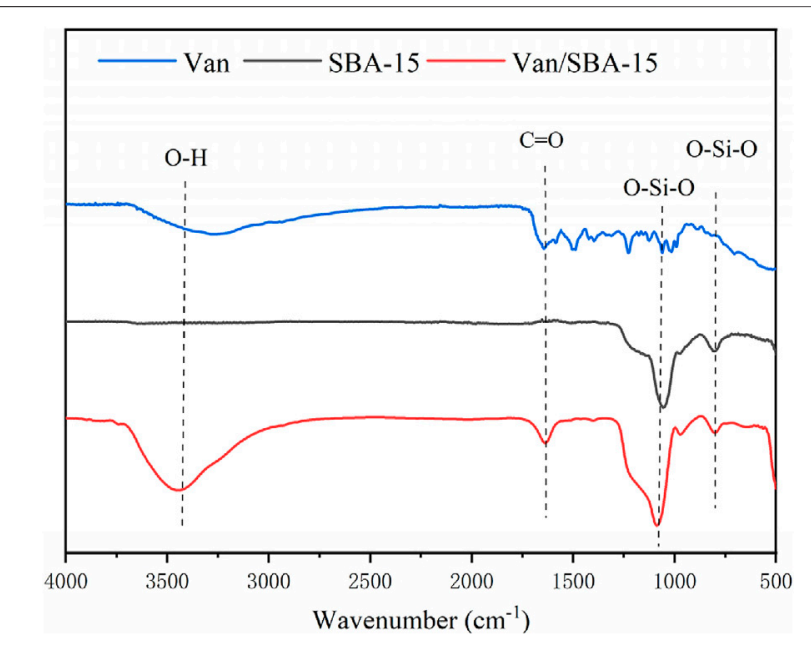

FIGURE 3 | FTIR spectra of Van, SBA-15, and Van/SBA-15.



while N1s and Cl2p peak did not appear in SBA-15 powder (Figure 2). The XPS results prove from the side that Van is successfully loaded in SBA-15.
The FTIR spectrum of Van showed peaks at $3,440 \mathrm{~cm}^{-1}$ and $1,635 \mathrm{~cm}^{-1}$, which corresponded to the $-\mathrm{OH}$ stretching and the $\mathrm{C}=\mathrm{O}$ vibration, respectively. These peaks are the characteristic peaks of Van. Furthermore, characteristic peaks corresponding to the stretching and bending of Si-O-Si in SBA-15 appeared at $1,090 \mathrm{~cm}^{-1}$ and $795 \mathrm{~cm}^{-1}$ (Kayan, 2016). The FTIR spectrum of Van/SBA-15 showed all the characteristic peaks of Van and SBA15 , confirming that Van was successfully encapsulated in SBA-15 (Figure 3).

The $\mathrm{N}_{2}$ adsorption-desorption isotherms before and after drug loading conformed to the type IV adsorption curve, which is the typical adsorption isotherms of mesoporous materials. The $\mathrm{N}_{2}$ adsorption after drug loading was significantly lower than that before drug loading, and both exhibited adsorption isotherm type IV and contained hysteresis loop (Figure 4). From the data given in Table 1, the loading of drug into the mesoporous pores of the material had no effect on the skeletal structure of SBA-15 but caused the reduction of pore size, pore volume, and specific surface area. This indicates that Van successfully entered into the pore channels of SBA-15 but not completely filled the pore channels.

\subsection{Characteristics of Van/SBA-15/ CS-GP-SA}

The SEM image showed that samples in all the 3 groups had distinct porous structures. The pore walls of CS-GP-SA were smooth. When Van was added, the pore walls of the prepared Van/CS-GP-SA became rough, and the surface showed a protruding granularity. With the addition of Van/SBA-15, the pore walls of the prepared Van/SBA-15/CS-GP-SA were rougher, and the surface presented a more pronounced granularity. Similarly, we found that the addition of Van and Van/SBA-15 affected the pore size of the scaffolds. The pore size of the manufactured Van/SBA-15/CS-GP-SA was larger than the other two groups (Figure 5). This indicates that the addition of Van/SBA-15 to CS-GP-SA has a significant influence on the surface structure (Jaidev and Chatterjee, 2019; Ouyang et al., 2019; Metwally et al., 2020; Wychowański et al., 2021).

\subsection{In Vitro Drug Release}

The controlled release curves of Van/SBA-15, Van/CS-GP-SA, and Van/SBA-15/CS-GP-SA showed that Van initiated burst release within the first $4 \mathrm{~d}$ and then sustained release 
TABLE 1 | Pore parameters of SBA-15 and Van/SBA-15.

\begin{tabular}{lcc} 
Sample & $\begin{array}{c}\text { BJH adsorption average } \\
\text { pore diameter }\end{array}$ & $\begin{array}{c}\text { BJH adsorption cumulative } \\
\text { volume of pores }\end{array}$ \\
\hline SBA-15 & $10.6 \mathrm{~m}$ & $1.1 \mathrm{~cm}^{3} / \mathrm{g}$ \\
Van/SBA-15 & $10.2 \mathrm{~nm}$ & $0.9 \mathrm{~cm}^{3} / \mathrm{g}$
\end{tabular}
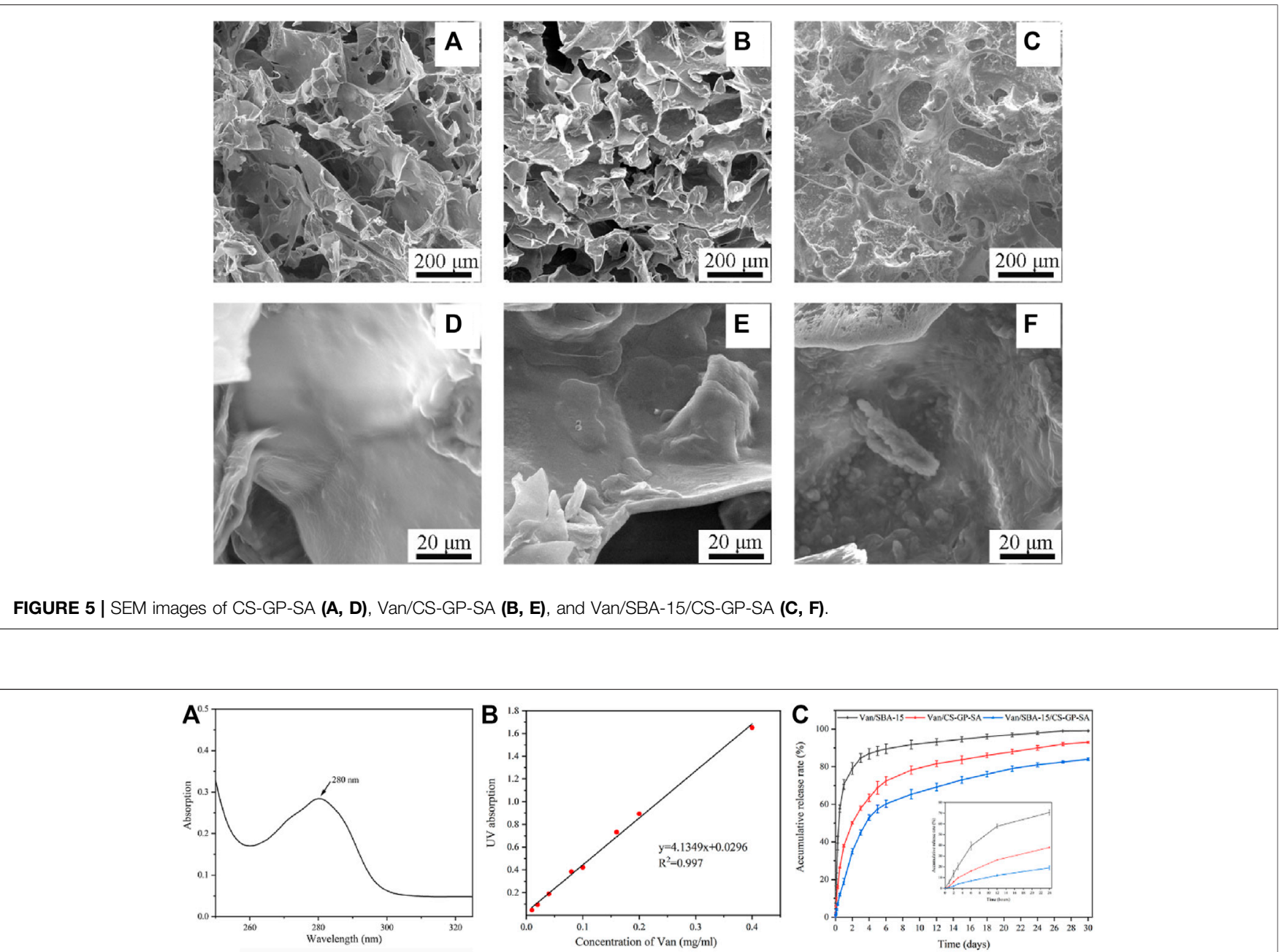

FIGURE 6 | Ultraviolet absorption of Van solution (A), the standard curve of Van solution (B), the cumulative release profiles of Van from Van/SBA-15, Van/CS-GPSA, and Van/SBA-15/CS-GP-SA (C).

thereafter. However, the slower release was observed in Van/ CS-GP-SA and Van/SBA-15/CS-GP-SA. The cumulative release of Van from Van/SBA-15, Van/CS-GP-SA, and Van/ SBA-15/CS-GP-SA on day 15 was $(94.6 \pm 1.37) \%$, (83.7 \pm $2.10) \%$, and $(73 \pm 1.76) \%$, respectively, and that on day 30 was $(99.1 \pm 0.13) \%,(93 \pm 0.47) \%$, and $(84 \pm 0.64) \%$, respectively (Figure 6). These results suggest that the sustained-release of Van/SBA-15/CS-GP-SA was much better than those in other samples, which is probably due to the drug combined with nanoparticles by hydrogen bonding, ionic interaction, and physical absorption (Wang et al., 2021a).

\subsection{In Vitro Cytocompatibility}

The CCK-8 data of all the four groups observed on 1,3 , and $5 \mathrm{~d}$ were not significantly different $(p>0.05)$. In addition, the cell proliferation indicated by the optical density (OD) values in all four groups increased with time $(p<0.05)$ (Figure 7A). The result was again confirmed with the nuclear staining method using DAPI for 1, 3, and 5 d. Figure 7B shows that all groups achieved a gradual increase in cell numbers with increasing incubation time, and there was no significant difference between the groups. The results indicate that the prepared composite hydrogels have good cytocompatibility and allow cell spreading and proliferation. The 

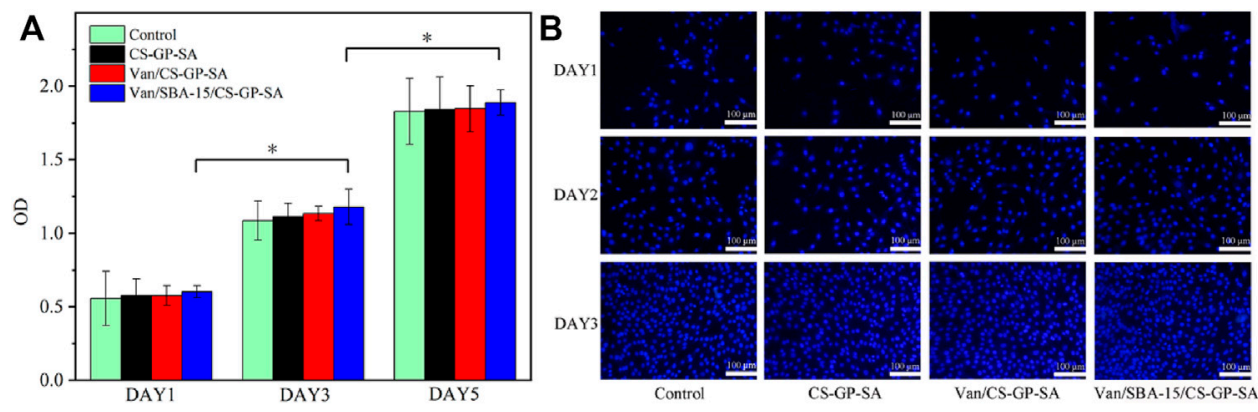

FIGURE 7 | CCK-8 data (A) and DAPI staining (B) of control, CS-GP-SA, Van/CS-GP-SA, and Van/SBA-15/CS-GP-SA.
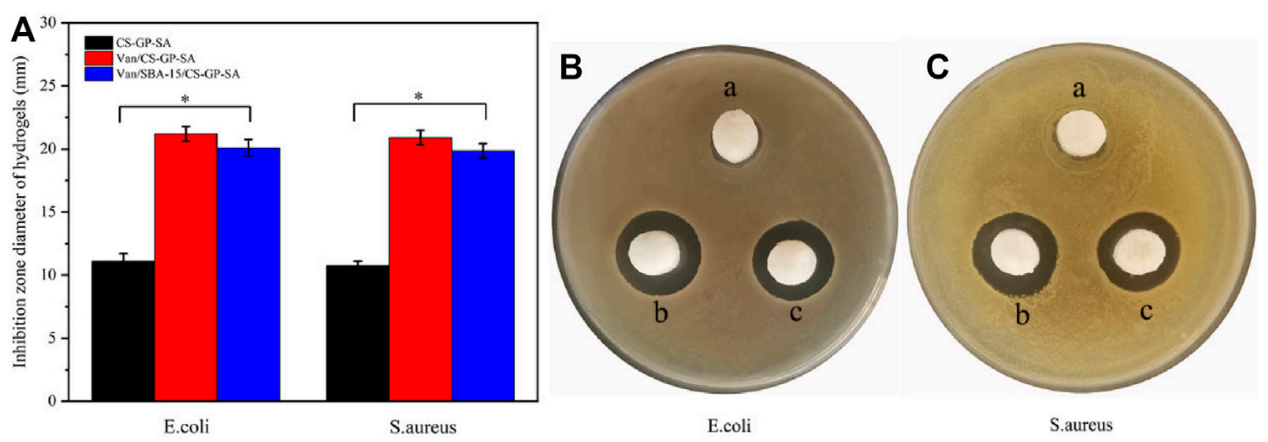

FIGURE 8 | Quantitative evaluation of in vitro antibacterial effect. (A) Antibacterial test results for E. coli (B) and S. aureus (C) of CS-GP-SA (a), Van/CS-GP-SA (b), and Van/SBA-15/CS-GP-SA (c).

above results confirm that Van/SBA-15/CS-GP-SA has high cytocompatibility.

\subsection{In Vitro Antibacterial Effect}

The bacteriostatic circle was more obvious in Van/SBA-15/CSGP-SA and Van/CS-GP-SA than that in CS-GP-SA $(p<0.05)$. A less pronounced antimicrobial cyclic appeared around CSGP-SA, and this may be caused by the antibacterial activity of CS (Zhu et al., 2021) (Figures 8B,C). These results indicate that Van/SBA-15/CS-GP-SA has excellent antibacterial properties.

However, as can be seen in the bar chart, Van/CS-GP-SA was slightly better than Van/SBA-15/CS-GP-SA, and this may be due to the slow release of SBA-15 (Figure 8A). The aformentioned finding further confirms that Van/SBA-15/CS-GP-SA has a sustained-release ability.

\section{CONCLUSION}

In this study, a novel temperature-sensitive hydrogel-based dual sustained-release system (Van/SBA-15/CS-GP-SA) was successfully prepared by Van-loaded SBA-15 and chitosan-glycerophosphate-alginate-sodium hydrogel, which have promising sustained-release ability, excellent biocompatibility, and antibacterial property. This study provides ideas for exploring new strategies for the management of infectious jaw defects.

\section{DATA AVAILABILITY STATEMENT}

The raw data supporting the conclusion of this article will be made available by the authors, without undue reservation.

\section{AUTHOR CONTRIBUTIONS}

MS contributed to investigation, data curation, visualization, and writing-original draft. LC helped with visualization and writing-original draft. $\mathrm{YX}$ and $\mathrm{XZ}$ assissted with investigation and data curation. DZ and ZX helped with software. LC and YL contributed to conceptualization, methodology, resources, and writing-review and editing. JS and QZ involved in conceptualization, methodology, and resources.

\section{FUNDING}

This study was supported by Shandong Medical and HealthScience and Technology Development Plan Project (2016WS0179). 


\section{REFERENCES}

Abid, S., Hussain, T., Nazir, A., Zahir, A., Ramakrishna, S., Hameed, M., et al. (2019). Enhanced Antibacterial Activity of PEO-Chitosan Nanofibers with Potential Application in Burn Infection Management. Int. J. Biol. Macromolecules 135, 1222-1236. doi:10.1016/j.ijbiomac.2019.06.022

Abinaya, B., Prasith, T. P., Ashwin, B., Viji Chandran, S., and Selvamurugan, N. (2019). Chitosan in Surface Modification for Bone Tissue Engineering Applications. Biotechnol. J. 14, 1900171. doi:10.1002/biot.201900171

Ahadi, F., Khorshidi, S., and Karkhaneh, A. (2019). A Hydrogel/fiber Scaffold Based on Silk Fibroin/oxidized Pectin with Sustainable Release of Vancomycin Hydrochloride. Eur. Polym. J. 118, 265-274. doi:10.1016/j.eurpolymj.2019. 06.001

Alazzawi, H. F., Salih, I. K., and Albayati, T. M. (2021). Drug Delivery of Amoxicillin Molecule as a Suggested Treatment for Covid-19 Implementing Functionalized Mesoporous SBA-15 with Aminopropyl Groups. Drug Deliv. 28, 856-864. doi:10.1080/10717544.2021.1914778

Alkafajy, A. M., and Albayati, T. M. (2020). High Performance of Magnetic Mesoporous Modification for Loading and Release of Meloxicam in Drug Delivery Implementation. Mater. Today Commun. 23, 100890. doi:10.1016/j. mtcomm.2019.100890

Bavnhøj, C. G., Knopp, M. M., Madsen, C. M., and Löbmann, K. (2019). The Role Interplay between Mesoporous Silica Pore Volume and Surface Area and Their Effect on Drug Loading Capacity. Int. J. Pharmaceutics: X 1, 100008. doi:10. 1016/j.ijpx.2019.100008

Cui, C., Sun, S., Wu, S., Chen, S., Ma, J., and Zhou, F. (2021). Electrospun Chitosan Nanofibers for Wound Healing Application. Engineered Regen. 2, 82-90. doi:10. 1016/j.engreg.2021.08.001

Darwich, K., Ismail, M. B., Al-Mozaiek, M. Y. A.-S., and Alhelwani, A. (2021). Reconstruction of Mandible Using a Computer-Designed 3D-Printed Patientspecific Titanium Implant: a Case Report. Oral Maxillofac. Surg. 25, 103-111. doi:10.1007/s10006-020-00889-w

Esperanza Adrover, M., Pedernera, M., Bonne, M., Lebeau, B., Bucalá, V., and Gallo, L. (2020). Synthesis and Characterization of Mesoporous SBA-15 and SBA-16 as Carriers to Improve Albendazole Dissolution Rate. Saudi Pharm. J. 28, 15-24. doi:10.1016/j.jsps.2019.11.002

Han, W., Zhang, L., Yu, L. j., and Wang, J. q. (2021). Effect of Local Delivery of Vancomycin and Tobramycin on Bone Regeneration. Orthop. Surg. 13, 1654-1661. doi:10.1111/os.13020

Hao, Y., Zheng, W., Sun, Z., Zhang, D., Sui, K., Shen, P., et al. (2021). Marine Polysaccharide-Based Composite Hydrogels Containing Fucoidan: Preparation, Physicochemical Characterization, and Biocompatible Evaluation. Int. J. Biol. Macromolecules 183, 1978-1986. doi:10.1016/j. ijbiomac.2021.05.190

Jaidev, L. R., and Chatterjee, K. (2019). Surface Functionalization of 3D Printed Polymer Scaffolds to Augment Stem Cell Response. Mater. Des. 161, 44-54. doi:10.1016/j.matdes.2018.11.018

Ji, Y., Han, Z., Ding, H., Xu, X., Wang, D., Zhu, Y., et al. (2021). Enhanced Eradication of Bacterial/Fungi Biofilms by Glucose Oxidase-Modified Magnetic Nanoparticles as a Potential Treatment for Persistent Endodontic Infections. ACS Appl. Mater. Inter. 13, 17289-17299. doi:10.1021/acsami.1c01748

Kang, M. S., Kang, J. I., Le Thi, P., Park, K. M., Hong, S. W., Choi, Y. S., et al. (2021). Three-Dimensional Printable Gelatin Hydrogels Incorporating Graphene Oxide to Enable Spontaneous Myogenic Differentiation. ACS Macro Lett. 10, 426-432. doi:10.1021/acsmacrolett.0c00845

Kayan, A. (2016). Synthesis, Characterization, and Application of Hybrid Inorganic-Organic Composites $(\mathrm{K} / \mathrm{Na}) \mathrm{ZrSi}(\mathrm{R}) \mathrm{Ox}$. J. Inorg. Organomet. Polym. 26, 640-647. doi:10.1007/s10904-016-0349-z

Kayan, G. Ö., and Kayan, A. (2021). Composite of Natural Polymers and Their Adsorbent Properties on the Dyes and Heavy Metal Ions. J. Polym. Environ. 29, 3477-3496. doi:10.1007/s10924-021-02154-x

Khorasani, H. R., Sanchouli, M., Mehrani, J., and Sabour, D. (2021). Potential of Bone-Marrow-Derived Mesenchymal Stem Cells for Maxillofacial and Periodontal Regeneration: A Narrative Review. Int. J. Dentistry, 2021, 1-13. doi:10.1155/2021/4759492

Kiti, K., and Suwantong, O. (2020). Bilayer Wound Dressing Based on Sodium Alginate Incorporated with Curcumin- $\beta$-Cyclodextrin Inclusion Complex/ chitosan Hydrogel. Int. J. Biol. Macromolecules 164, 4113-4124. doi:10.1016/ j.ijbiomac.2020.09.013

Laurano, R., Boffito, M., and Martino, P. (2020). Thermosensitive Micellar Hydrogels as Vehicles to Deliver Drugs With Different Wettability. Front. Bioeng. Biotechnol. 8, 708. doi:10.3389/fbioe.2020.00708

Li, Y., Chi, Y. Q., Yu, C. H., Xie, Y., Xia, M. Y., Zhang, C. L., et al. (2020). Drug-free and Non-crosslinked Chitosan Scaffolds with Efficient Antibacterial Activity against Both Gram-Negative and Gram-Positive Bacteria. Carbohydr. Polym. 241, 116386. doi:10.1016/j.carbpol.2020.116386

Ma, Y., Shi, L., Liu, F., Zhang, Y., Pang, Y., and Shen, X. (2019). Self-assembled Thixotropic Silver Cluster Hydrogel for Anticancer Drug Release. Chem. Eng. J. 362, 650-657. doi:10.1016/j.cej.2019.01.096

Metwally, S., Ferraris, S., Spriano, S., Krysiak, Z. J., Kaniuk, Ł., Marzec, M. M., et al. (2020). Surface Potential and Roughness Controlled Cell Adhesion and Collagen Formation in Electrospun PCL Fibers for Bone Regeneration. Mater. Des. 194, 108915. doi:10.1016/j.matdes.2020.108915

Moritz, M., and Łaniecki, M. (2012). Application of SBA-15 Mesoporous Material as the Carrier for Drug Formulation Systems. Papaverine Hydrochloride Adsorption and Release Study. Powder Tech. 230, 106-111. doi:10.1016/j. powtec.2012.06.061

Mujtaba, M. A., and Alotaibi, N. M. (2020). Chitosan-sodium Alginate Nanoparticle as a Promising Approach for Oral Delivery of Rosuvastatin Calcium: Formulation, Optimization and In Vitro Characterization. Jpri 32, 50-56. doi:10.9734/jpri/2020/v32i130394

Op 't Veld, R. C., Eerden, M., Wagener, F. A. D. T. G., Kouwer, P. H. J., Jansen, J. A., Walboomers, X. F., et al. (2020). Polyisocyanopeptide Hydrogels Are Effectively Sterilized Using Supercritical Carbon Dioxide. Tissue Eng. C: Methods 26, 132-141. doi:10.1089/ten.TEC.2019.0305

Ouyang, P., Dong, H., He, X., Cai, X., Wang, Y., Li, J., et al. (2019). Hydromechanical Mechanism behind the Effect of Pore Size of Porous Titanium Scaffolds on Osteoblast Response and Bone Ingrowth. Mater. Des. 183, 108151. doi:10.1016/j.matdes.2019.108151

Pecci, R., Baiguera, S., Ioppolo, P., Bedini, R., and Del Gaudio, C. (2020). 3D Printed Scaffolds with Random Microarchitecture for Bone Tissue Engineering Applications: Manufacturing and Characterization. J. Mech. Behav. Biomed. Mater. 103, 103583. doi:10.1016/j.jmbbm.2019.103583

Pierozan, P., and Karlsson, O. (2019). Mitotically Heritable Effects of BMAA on Striatal Neural Stem Cell Proliferation and Differentiation. Cell Death Dis 10, 478. doi:10.1038/s41419-019-1710-2

Seljak, K. B., Kocbek, P., and Gašperlin, M. (2020). Mesoporous Silica Nanoparticles as Delivery Carriers: An Overview of Drug Loading Techniques. J. Drug Deliv. Sci. Tech. 59, 101906. doi:10.1016/j.jddst.2020. 101906

Shen, Z., Cai, N., Xue, Y., Yu, B., Wang, J., Song, H., et al. (2020). Porous SBA-15/ cellulose Membrane with Prolonged Anti-microbial Drug Release Characteristics for Potential Wound Dressing Application. Cellulose 27, 2737-2756. doi:10.1007/s10570-020-02967-4

Sordi, M. B., Cruz, A., Fredel, M. C., Magini, R., and Sharpe, P. T. (2021). Threedimensional Bioactive Hydrogel-Based Scaffolds for Bone Regeneration in Implant Dentistry. Mater. Sci. Eng. C 124, 112055. doi:10.1016/j.msec.2021. 112055

Sun, J. G., Jiang, Q., Zhang, X. P., Shan, K., Liu, B. H., Zhao, C., et al. (2019). Mesoporous Silica Nanoparticles as a Delivery System for Improving Antiangiogenic Therapy. Ijn 14, 1489-1501. doi:10.2147/IJN.S195504

Vestby, L. K., Grønseth, T., Simm, R., and Nesse, L. L. (2020). Bacterial Biofilm and its Role in the Pathogenesis of Disease. Antibiotics 9, 59. doi:10.3390/ antibiotics9020059

Wang, X., Fang, L., Wang, S., Chen, Y., Ma, H., Zhao, H., et al. (2020). Antibiotic Treatment Regimens for Bone Infection after Debridement: A Study of 902 Cases. BMC Musculoskelet. Disord. 21, 1-6. doi:10.1186/ s12891-020-03214-4

Wang, Z., Mei, L., Liu, X., and Zhou, Q. (2021a). Hierarchically Hybrid Biocoatings on Ti Implants for Enhanced Antibacterial Activity and Osteogenesis. Colloids Surf. B: Biointerfaces 204, 111802. doi:10.1016/j.colsurfb.2021.111802

Wang, Z., Wang, X., Wang, Y., Zhu, Y., Liu, X., and Zhou, Q. (2021b). NanoZnOmodified Titanium Implants for Enhanced Anti-bacterial Activity, Osteogenesis and Corrosion Resistance. J. Nanobiotechnol 19, 1-23. doi:10. 1186/s12951-021-01099-6 
Wychowański, P., Starzyńska, A., Adamska, P., Słupecka-Ziemilska, M., Sobocki, B. K., Chmielewska, A., et al. (2021). Methods of Topical Administration of Drugs and Biological Active Substances for Dental Implants-A Narrative Review. Antibiotics 10, 919-921. doi:10.3390/antibiotics10080919

Yang, L., Pijuan-Galito, S., Rho, H. S., Vasilevich, A. S., Eren, A. D., Ge, L., et al. (2021). High-Throughput Methods in the Discovery and Study of Biomaterials and Materiobiology. Chem. Rev. 121, 4561-4677. doi:10.1021/acs.chemrev. $0 \mathrm{c} 00752$

Yin, X., Hao, Y., Lu, Y., Zhang, D., Zhao, Y., Mei, L., et al. (2021). BioMultifunctional Hydrogel Patches for Repairing Full-Thickness Abdominal Wall Defects. Adv. Funct. Mater, 31, 2105614. doi:10.1002/ adfm.202105614

Zhang, Q., Wu, W., Qian, C., Xiao, W., Zhu, H., Guo, J., et al. (2019). Advanced Biomaterials for Repairing and Reconstruction of Mandibular Defects. Mater. Sci. Eng. C 103, 109858. doi:10.1016/j.msec.2019.109858

Zhao, Z., Vizetto-Duarte, C., Moay, Z. K., Setyawati, M. I., Rakshit, M., Kathawala, M. H., et al. (2020). Composite Hydrogels in Three-Dimensional In Vitro Models. Front. Bioeng. Biotechnol. 8, 1-22. doi:10.3389/fbioe.2020.00611

Zheng, W., Hao, Y., Wang, D., Huang, H., Guo, F., Sun, Z., et al. (2021). Preparation of Triamcinolone Acetonide-Loaded Chitosan/fucoidan Hydrogel and its Potential Application as an Oral Mucosa Patch. Carbohydr. Polym. 272, 118493. doi:10.1016/j.carbpol.2021.118493
Zhu, Y., Liu, L., Sun, Z., Ji, Y., Wang, D., Mei, L., et al. (2021). Fucoidan as a marine-origin Prebiotic Modulates the Growth and Antibacterial Ability of Lactobacillus Rhamnosus. Int. J. Biol. Macromolecules 180, 599-607. doi:10. 1016/j.ijbiomac.2021.03.065

Conflict of Interest: The authors declare that the research was conducted in the absence of any commercial or financial relationships that could be construed as a potential conflict of interest.

Publisher's Note: All claims expressed in this article are solely those of the authors and do not necessarily represent those of their affiliated organizations, or those of the publisher, the editors, and the reviewers. Any product that may be evaluated in this article, or claim that may be made by its manufacturer, is not guaranteed or endorsed by the publisher.

Copyright $\odot 2022$ Sun, Cheng, Xu, Chen, Liu, Xu, Zhou, Zhang, Zhou and Sun. This is an open-access article distributed under the terms of the Creative Commons Attribution License (CC BY). The use, distribution or reproduction in other forums is permitted, provided the original author(s) and the copyright owner(s) are credited and that the original publication in this journal is cited, in accordance with accepted academic practice. No use, distribution or reproduction is permitted which does not comply with these terms. 